\title{
NÉCROSE PANCRÉATIQUE INFECTIEUSE DES SALMONIDÉS : ÉVALUATION DE MÉTHODES DESTINÉES À COUPÉR LA TRANSMISSION PAR L'OEUF.
}

\author{
M. DÓRSON (1), C. TORHY (1), R. BILLARD (2), C. SAUDRAIS (3), G. MAISSE (3), \\ P. HAFFRAY (4), M.G. HOLLEBECQ (5).
}

1) Laboratoire de Virologie et Immunologie Moléculaires, Pathologie infectieuse et Immunité des Poissons, INRA, Domaine de Vilvert, 78352 Jouy=en-Josas Cedex, France.

2) Laboratoire d'Ichtyologie, Muséum National d'Histoire Naturelle, 43 rue Cuvier, 75231 Paris, France.

3) Laboratoire de Physiologie des Poissons, INRA, Campus de Beaulieu, 35042 Rennes, France.

4) Syndicat des Sélectionneurs Avicoles et Aquacoles Français, Complexe Agronomique, 65 rue, de Saint-Brieuc, 35042 Rennes, France.

5) Laboratoire de Génétique des Poissons, INRA, Domaine de Vilvert, 78352 Jouy-enJosas Cedex, France.

Reçu le 23 février 1995

Accepté le 11 octobre 1995

Received 23 February, 1995

Accepted 11 October, 1995

\section{RÉSUMÉ}

Des ovules de truites Arc-en-Ciel ont été fécondés par des spermatozoïdes de la même espèce traités par l'iode suivant différents protocoles afin de couper la transmission de la nécrose pancréatique infectieuse (NPI) des salmonidés. L'efficacité de l'iọde a été évaluée en parallèle par neutralisation du virus en $1 \mathrm{mn}$. Le protocole conseillé pour la solution d'iode disponible dans le commerce (ovules égouttés, puis recouverts de solution iodée et sperme ajouté immédiatement) a d'abord été utilisé. A pH compris entre 5 et 8 et pour des concentrations en iode de 15,25 et $35 \mathrm{mg} / \mathrm{l}$, le pourcentage de fécondation est inférieur à 10 et ne remonte qu'à $\mathrm{pH} 9(69 \%$ pour $25 \mathrm{mg} / \mathrm{l})$. Par contre, l'efficacité contre le virus décroît rapidement pour des $\mathrm{pH}$ supérieurs à 7 , et aucun $\mathrm{pH}$ n'a permis de concilier efficacité et innocuité. Le même protocole utilisé avec 10 spermes individuels a montré des différences de sensibilité individuelle marquées (pourcentages de fécondation de 1,6 à $37 \%$ pour $25 \mathrm{mg} / \mathrm{l}$ d'iode à pH8), qui ne sont pas en corrélation avec la qualité des spermes appréciée par la concentration de la protéine de membrane $42 \mathrm{kDa}$ dans le liquide séminal. Les différents essais de préincubation du sperme (de quelques secondes à 1 minute) ont donné des rendements de fécondation nuls, sauf pour un large excès de sperme, qui entraînait aussi la perte de l'efficacité de l'iode contre le virus. Deux expériences de transmission expérimentale ont été réalisées en incubant les spermatozoïdes avec du virus concentré. L'élimination systématique des œufs morts et anormaux suivie d'une désinfection externe des œufs (50 $\mathrm{mg} / \mathrm{l}$ d'iode, $15 \mathrm{mn}$ ) avant l'éclosion n'a pas permis de couper la transmission, ce qui laisse supposer que le virus peut être transmis sans infecter l'embryon de façon létale.

\section{INFECTIOUS PANCREATIC NECROSIS OF SALMONIDS : ASSESSMENT OF METHODS AIMING AT CUTTING OFF EGG-ASSOCIATED TRANSMISSION.}

\section{SUMMARY}

Rainbow trout ova were fertilized by milt treated with iodine following different schedules, in order to cut off infectious pancreatic necrosis (IPN) transmission. The efficacy of iodine was assessed in parallel by virus neutralization during a $1 \mathrm{~min}$. exposure. The schedule recommended for the commercialized iodine solution (ova drained, covered with iodine solution and milt added immediately) was used first. When the $\mathrm{pH}$ ranged from 5 to 8 and for iodine concentrations of 15,25 , and $35 \mathrm{mg} / \mathrm{l}$, the fecundation rate was below $10 \%$ 
and rose only at $\mathrm{pH} 9$ (69\% for $25 \mathrm{mg} / \mathrm{l}$ iodine). In other hand, the efficacy against the virus decreased rapidly for $\mathrm{pH}$ values above 7 , and efficacy could never match with safety at any $\mathrm{pH}$. The same schedule used with 10 individual milts showed striking differences in individual susceptibilities (fecundation rates from 1.6 to $37 \%$ for $25 \mathrm{mg} / \mathrm{l}$ iodine at $\mathrm{pH} 8$ ) which did not correlate with the quality of the milt as estimated by the concentration of the $42 \mathrm{kDa}$ membrane protein in seminal fluid. Preincubation of milt from a few seconds to 1 minute in different concentrations of iodine resulted in null fecundation rates, except when milt was in large excess. In that case, iodine lost all efficacy against the virus. Two experimental transmission experiments were done by incubating sperms with concentrated virus. A careful elimination of dead and abnormal eggs followed by an external disinfection (50 $\mathrm{mg} / \mathrm{l}$ iodine, $15 \mathrm{~min}$.) before hatching did not allow to cut off transmission. This indicates that the virus can be transmitted without lethally infecting the embryo.

\section{INTRODUCTION}

La Nécrose Pancréatique Infectieuse (NPI) des salmonidés, d'abord décrite chez des alevins d'omble de fontaine (Salvelinus fontinalis Mitchill) par WOOD, SNIESZKO et YASUTAKE (1955) est caractérisée avant tout, comme son nom l'indique, par une nécrose importante du pancréas exocrine qui accompagne des lésions de la paroi du tube digestif (SNIESZKO, WOOD et YASUTAKE, 1957). Le virus responsable de la maladie fut isolé par WOLF, SNIESZKO, DUNBAR et PYLE en 1960, et classé dans la famille des birnaviridae (DOBOS, HILL, HALLETT, KELLS, BECHT et TENINGES, 1979). Ce virus qui présente un grand nombre de sérotypes pouvant posséder des pouvoirs pathogènes différents (HILL, 1982 ; WOLF, 1988) affecte avant tout plusieurs espèces de salmonidés, la truite Arc-enCiel (Oncorhynchus mykiss Walbaum) apparaissant parmi les plus sensibles, juste derrière l'omble de fontaine (SILIM, ELAZHARY et LAGACE, 1982). Des virus de la NPI ont été isolés dans tous les pays où sont élevés des salmonidés et leur ubiquité est un obstacle aux tentatives de prophylaxie sanitaire tandis que la vaccination apparaît peu utilisable en pratique (DORSON, 1988).

A de rares exceptions près, cette maladie n'atteint que des alevins âgés de moins de 1500 degrés X jours (FRANTSI et SAVAN, 1971 ; DORSON et TORCHY, 1981 ; LAPIERRE, LARRIVEE et BERTHIAUME, 1986). Une fraction importante des survivants est constituée de porteurs asymptomatiques, et il a été montré dès 1963 par WOLF, QUIMBY èt BRADFORD et confirmé par. FIJAN et GIORGETTI (1978) ainsi que par BOOTLAND, DOBOS et STEVENSON (1991) que la maladie pouvait être transmise par l'oeuf, puis en 1976 par BULLOCK, RUCKER, AMEND, WOLF et STUCKEY que cette transmission n'était pas coupée par la désinfection externe des oeufs à l'aide d'un iodophore à $50 \mathrm{mg} / \mathrm{l}$ d'iode actif. Or, cette concentration est largement suffisante pour inactiver le virus de la NPI (AMEND et PIETSCH, 1972 ; DESAUTELS et MACKELVIE, 1975 ; ELLIOT et AMEND, 1978). Dès 1963, l'iode solubilisé avec du polyvinylpyrrolidone et incorporé à la nourriture a été employé avec un succès partiel pour lutter contre la NPI par ECONOMON (1963). La constatation répétée que le virus peut être transmis des géniteurs aux alevins à l'intérieur de l'oeuf a suscité des travaux dans deux directions principales: préciser le mode d'infection des oeufs d'une part, tenter de couper la transmission à l'aide de désinfectants d'autre part. Il fut observé par AHNE (1983) et par CASTRIC (1984) que certaines truites mâles pouvaient émettre un sperme riche en particules virales, tandis que MULCAHY et PASCHO (1984) montraient que le virus pouvait s'adsorber aux spermatozoïdes. Il fut ensuite démontré par DORSON et TORCHY (1985) que l'infection du sperme in vitro était la seule manière sûre de réaliser la transmission expérimentale à l'intérieur de l'oeuf, tandis que la possibilité d'une transmission de surface - très facile à couper par désinfection - était confirmée par AHNE et NEGELE (1985). Plus récemment, RODRIGUEZ SAINT-JEAN, PEREZ PRIETO et VILAS MINONDO (1992) ont confirmé la fixation du virus de la NPI aux spermatozoïdes grâce à la cytométrie de flux qui peut permettre l'analyse rapide d'un grand nombre d'échantillons. L'intérêt des iodophores pour la désinfection externe des oeufs a été souligné par AMEND (1974) qui a défini les concentrations supportables par les oeufs pendant le durcissement, après celui-ci et au stade oeillé. Si la dose de $25 \mathrm{mg} / \mathrm{l}$ d'iode actif (limite pour une inactivation rapide du virus) était supportée durant $15 \mathrm{mn}$ à tous les stades, la dose "sécuritaire" de $100 \mathrm{mg} / \mathrm{ne}$ 
l'était qu'après un durcissement de $30 \mathrm{mn}$. Une dose moyenne de $50 \mathrm{mg} / \mathrm{l}$ a été employée par BILLARD et DE MONTALEMBERT (1976) pour traiter les gamètes de truite Arc-en-Ciel et les oeufs à divers stades. Si les ovules supportaient ce traitement, il n'en était pas de même pour les spermatozoïdes, totalement inactivés si la fécondation était pratiquée dans la solution iodée. Cependant, subsistait l'espoir d'utiliser la dose représentant le meilleur compromis «efficacité contre le virus :-'innocuité. pour les spermatozoïdes", 25 mg/l d'iode actif, et tandis que plusieurs équipes en étudiaient la possibilité, la méthode de fécondation en présence d'iode était décrite par KLONTZ (1981) et très vite préconisée dans la pratique, bien que son usage soit très vite apparu dangereux (DORSON et TORCHY, 1985) lorsque l'iode était dilué dans l'eau pure. De la même façon AHNE, KELLY et SCHLOTFELDT (1989) n'arrivaient pas à concilier efficacité et innocuité. La connaissance de différents résultats préliminaires comme des observations de terrain conduisaient donc à une mise en garde (DORSON, 1989) tandis qu'étaient menées les expériences décrites ici, qui avaient pour but de comparer l'efficacité et l'innocuité de différentes pratiques utilisant un iodophore, et de déterminer l'influence d'une élimination soigneuse de tous les oeufs avortant pendant l'incubation. L'hypothèse de départ dans ce dernier cas était que le virus qui pénètre adsorbé à la surface du spermatozoïde infecterait les premières cellules de l'embryon. Ce dernier avorterait donc rapidement, constituant une réserve de virions susceptibles d'être libérés lors de la rupture tardive de la coque de l'oeuf. Cette hypothèse était encouragée par l'observation de AHNE, KELLY et SCHLOTFELDT (1989) que les alevins anormaux lors de l'éclosion étaient plus fréquemment porteurs du virus, tandis que FIJAN et GIORGETTI (1978) ne donnaient pas de détails sur l'état exact des oeufs porteurs de virus:

\section{MATÉRIELS ET TECHNIQUES}

Gamètes : les ovules et le sperme ont été prélevés sur des truites Arc-en-Ciel élevées à la pisciculture expérimentale INRA de Gournay/Aronde (Oise), conservés sous glace et utilisés dans les heures suivant le prélèvement. Cet établissement est l'objet de contrôles virologiques réguliers, et indemne de tout virus connu. Les ovules de 3 à 6 femelles suivant les expériences étaient répartis en nombre égal dans les différents lots. Les spermes ont été utilisés soit individuellement, soit en pools de 5 à 7 mâles différents.

Solutions d'insémination : outre l'eau bidistillée et l'eau du robinet, ont été utilisés le dilueur d'insémination artificielle (DIA) mis au point par BILLARD (1977) et commercialisé sóus le nom dé, DIA.532 : glycine $0 ; 05 \mathrm{M}$, tris (2 amino 2 hydroxymethyl $1-3$ propane-diol) $0,02 \mathrm{M}$, NaCl $0,1 \mathrm{M}$, pH9, et des solutions de tétraborate de sodium $\mathrm{Na}_{2} \mathrm{~B}_{4} \mathrm{O}_{7}$ $\left(10 \mathrm{H}_{2} \mathrm{O}\right)$ ajustées au $\mathrm{PH}$ désiré à l'aide d'HCl $16 \mathrm{~N}$ et additionnées de $\mathrm{NaCl} \mathrm{O}, 1 \mathrm{M}$. L'iode était ajouté à ces solutions immédiatement avant emploi.

lodophore : La spécialité commercialisée sous le nom de «ROMEIOD» contient $5 \mathrm{~g} / \mathrm{l}$ d'iode total, ce qui correspond à un minimum de $3,5 \mathrm{~g} / \mathrm{l}$ d'iode libre (données fabricant), $7 \mathrm{~g} / \mathrm{l}$ d'iodure de potassium, $88 \mathrm{~g} / \mathrm{l}$ de Polyvidone (ou polyvinylpyrrolidone). La neutralisation de l'iode était pratiquée à l'aide d'une solution de thiosulfate de sodium $\mathrm{Na}_{2} \mathrm{~S}_{2} \mathrm{O}_{3}$ commercialisé sous l'appellation «neutralisant» utilisé en excès.

Fécondation artificielle : dans tous les cas le liquide coelomique était éliminé à l'aide d'une passoire avant pesée et répartition des ovules. Selon la technique préconisée sur l'étiquette du flacon de "ROMEIOD" (1992) la solution iodée à $5 \mathrm{ml} / \mathrm{l}$ (soit $25 \mathrm{mg} / \mathrm{l}$ d'iode) est déposée sur les ovules. Le sperme est ajouté immédiatement à raison de $10 \mathrm{ml}$ pour 1 I d'ovules, et le mélange réalisé rapidement par transvasement. Après $5 \mathrm{mn}$ les oeufs sont placés dans l'eau d'incubation.

Dans certains cas le sperme était mélangé au dilueur de fécondation juste avant d'en recouvrir les ovules, ou préincubé dans un dilueur de conservation contenant 2 g/l de $\mathrm{KCl}$ (BILLARD et JALABERT, 1974) avant d'être utilisé suivant le protocole de fécondation "classique". Suivant ce dernier, utilisé par la majorité des salmoniculteurs, le sperme est déposé sur les ovules égouttés qui sont ensuite recouverts d'eau ou du dilueur trisglycocolle décrit plus haut et disponible dans le commerce. 
Incubation et élevage : les oeufs non infectés étaient incubés dans des incubateurs de $10 \times 10 \mathrm{~cm}$ placés dans des auges alimentées en eau recyclée et filtrée maintenue à $10^{\circ} \mathrm{C}$. Les expériences impliquant le virus étaient réalisées dans des aquariums de 10 I en circuit ouvert alimentés en eau de ville déchlorée sur charbon actif et thermorégulée à $10^{\circ} \mathrm{C}$.

Production, concentration et titrage du virus de la N.P.I. : Le virus de la N.P.I. souche 31.75 isolée à partir d'alevins de truite Arc-en-Ciel malades, et entretenue régulièrement sur alevins sensibles, a été produit sur cellules de truites RTG 2 (WOLF et QUIMBY, 1962) cultivées à $14^{\circ} \mathrm{C}$ en milieu de Stoker tamponné à $\mathrm{pH} 7,4$ par du tris- $\mathrm{HCl}$ $0,16 \mathrm{M}$, et supplémenté par du sérum fétal bovin à $2 \%$, et des antibiotiques (pénicilline 100 $\mathrm{Ul} / \mathrm{ml}$, streptomycine $0,1 \mathrm{mg} / \mathrm{ml}$, kanamycine $0,1 \mathrm{mg} / \mathrm{ml}$ ). Les cellules étaient infectées à raison d'environ une unité formant plage (u.f.p.) par cellule. Après destruction totale du tapis cellulaire le milieu de culture était clarifié par centrifugation $(5000 \mathrm{~g}, 15 \mathrm{mn})$ et additionné de Polyéthylène glycol (PEG, Carbowax 6000 ) à raison de $10 \%$ sous agitation. Après une nuit à $4{ }^{\circ} \mathrm{C}$ la suspension était centrifugée $(5000 \mathrm{~g}, 30 \mathrm{mn})$, et le culot repris dans environ $1 / 20$ du volume initial, dans une solution tamponnée à $\mathrm{pH} 6,4$, avec agitation vigoureuse et dissociation par les ultrasons (Bransonic, $200 \mathrm{~W}, 10$ kilocycles, $2 \mathrm{mn}$ à $0^{\circ} \mathrm{C}$ ). Le virus était titré par la technique des plages sous agarose décrite par DE KINKELIN et SCHERRER (1970): 0,1 ml de la suspension à titrer était déposé sur des tapis de cellules de $35 \mathrm{~mm}$ de diamètre (P6,Nunc). Après adsorption ( $1 \mathrm{~h}$ ), les cellules étaient recouvertes de milieu de culture additionné d'agarose à $0,4 \%$. Après $3 \mathrm{j}$ d'incubation à $14{ }^{\circ} \mathrm{C}$ les cellules étaient colorées par le rouge neutre et les plages de lyse dénombrées. Les poissons soumis au diagnostic virologique étaient broyés au mortier en présence de sable. Le broyat, repris en milieu de culture additionné d'antibiotiques, était clarifié par centrifugation $\left(5000 \mathrm{~g}, 15 \mathrm{mn}\right.$ ) et le surnageant inoculé à des tapis de cellules $\mathrm{RTG}_{2}$ réparties $24 \mathrm{~h}$ auparavant dans des plaques à 24 puits ( $\mathrm{P} 24, \mathrm{Nunc})$.

Inactivation du virus par l'iode : Une suspension de virus de la NPI concentrée au PEG et ramenée à un titre de $10^{7}$ u.f.p./ml par dilution dans une solution de $\mathrm{NaCl} 0,1 \mathrm{M}$ non tamponnée était diluée $(0,1 \mathrm{ml}$ dans $0,9 \mathrm{ml})$ dans la solution iodée ou non et avec une agitation immédiate. Après incubation $(1 \mathrm{mn})$ à la température ambiante l'iode était neutralisé par le thiosulfate (solution commerciale à $220 \mathrm{~g} / \mathrm{l}$ ) en excès, et le virus résiduel titré. II avait été contrôlé que la solution d'iode ainsi neutralisée n'avait pas d'influence sur la sensibilité des cellules au virus selon le protocole utilisé comme cela avait été évoqué par AMEND et PIETSCH (1972).

Titrage de la protéine de membrane $42 \mathrm{kDa}$ : Le pourcentage de protéine $42 \mathrm{kDa}$ par rapport aux protéines totales du fluide séminal a été déterminé selon la méthode décrite par MAISSE, PINSON et LOIR (1988).

Analyse statistique : Les coefficients de concordance entre les rangs de FRIEDMANN-KENDALL (SPRENT, 1992) ont été utilisés pour comparer les taux de fécondation avec ou sans iode et les critères de qualité du sperme.

\section{RÉSULTATS}

\section{Rendement des fécondations pratiquées suivant le protocole conseillé sur les flacons de «Romeiod»}

Les différents lots d'ovules $(n=196$ à $237, M=223$ ) ont été inséminés en utilisant le même volume d'un mélange de spermes, en faisant varier le $\mathrm{pH}$ et la concentration en iode de la solution utilisée pour activer les spermatozoïdes. Le rendement, apprécié en pourcentage d'oeufs présentant des vésicules optiques normales à $220^{\circ} \mathrm{X}$ jours, était de 97,3 pour le lot témoin «DIA» inséminé en début d'expérience et de 92,5 pour le lot témoin "DIA» de fin d'expérience (Fig. 1). En utilisant le milieu salin tamponné par le tétraborate à $\mathrm{pH}$ 7-8-9 les rendements sont restés dans cette fourchette, pour descendre à $60 \%$ à pH 6,3. Par contre, dès que le milieu de fécondation est additionné d'iode à $15 \mathrm{mg} / \mathrm{l}$ les rendements chutent aux alentours de $10 \%$ entre $\mathrm{pH}$ 5,3 (solution iodée non tamponnée en eau distillée) et $\mathrm{pH} 8$. Aux doses d'iode de 25 et $35 \mathrm{mg} / \mathrm{l}$ les rendements sont encore inférieurs, et ne remontent qu'à pH 9 (69\% pour $25 \mathrm{mg} / \mathrm{l})$. 


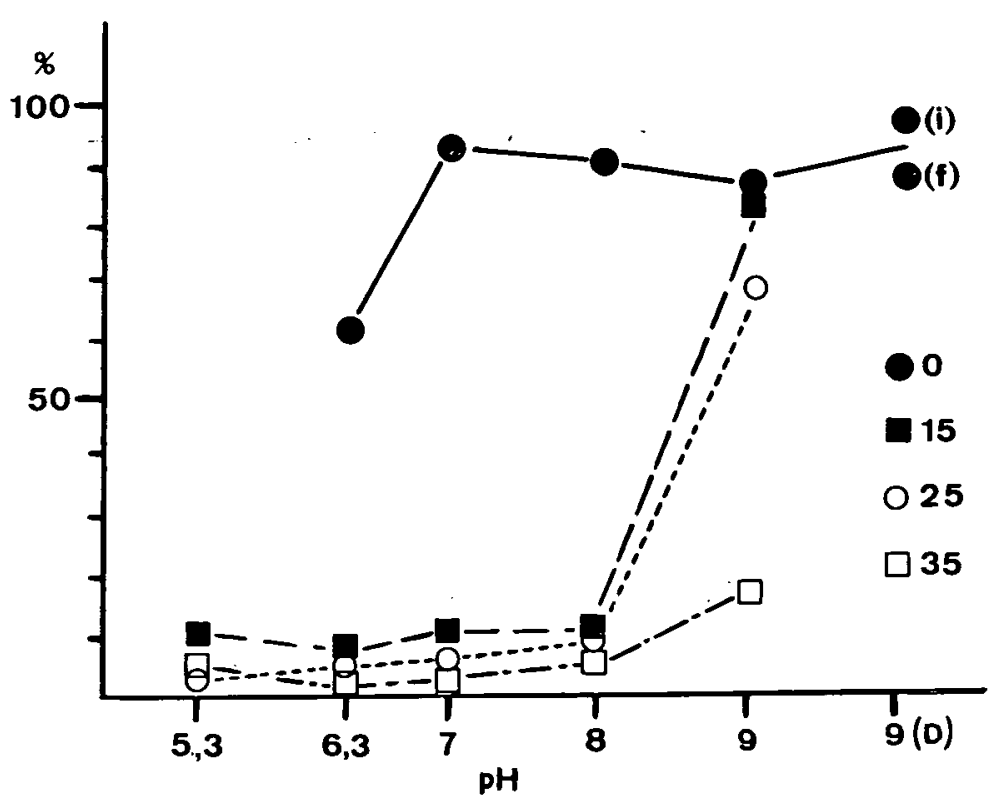

Figure 1 : Rendements de fécondation (en \% d'oeufs embryonnés) obtenus avec la technique de fécondation suivante : 220 ovules égouttés étaient recouverts par $30 \mathrm{ml}$ de milieu de fécondation. Le sperme $(0,3 \mathrm{ml})$ était ajouté immédiatement et le mélange effectué par transvasement. Les milieux de fécondation à pH 5,3 (eau distillée), 6,3,7,8 et 9 (tampon au tétraborate de sodium) contenaient $0,15,25$, et $35 \mathrm{mg} / \mathrm{l}$ d'iode actif. Un lot témoin de rendement maximum ( $D$, dilueur d'insémination artificielle tamponné à pH 9 au tris-glycine) a été inséminé au début (i) et à la fin (f) de la série.

Figure 1 : Fecundation rates obtained with the following fecundation schedule : 220 drained ova were covered with $30 \mathrm{ml}$ of the fecundation medium. Milt $(0.3 \mathrm{ml})$ was added immediately and rapidly mixed. The fecundation media at $\mathrm{pH} 5.3$ (distilled water), $6.3 ; 7,8$ and 9 (buffered with sodium tetraborate) contained $0,15,25$, and $35 \mathrm{mg} / \mathrm{l}$ of iodine. Control groups of maximum fecundation rate (D, commercial fecundation medium buffered at $\mathrm{pH} 9$ by tris-glycine) were inseminated at the beginning (i) and at the end $(f)$ of the series.

\section{Efficacité de l'iode in vitro sur le virus de la N.P.I.}

Après incubation $1 \mathrm{mn}$ dans la solution iodée et neutralisation de l'iode par le thiosulfate, le virus était titré, et l'inactivation appréciée par rapport à une suspension virale témoin. Les résultats indiquent (fig. 2) une inactivation totale de la suspension virale à pH 7 dès que la concentration en iode atteint $12 \mathrm{mg} / \mathrm{l}$. A pH 8, l'inactivation du virus n'est pas totale $(98 \%)$ même à la dose de $35 \mathrm{mg} / \mathrm{l}$ et n'atteint que $88 \%$ à $\mathrm{pH} 9$.

\section{Evaluation simultanée de l'innocuité et de l'efficacité d'une préparation d'iode à $25 \mathrm{mg} / \mathrm{l}$}

Les protocoles utilisés ont été les mêmes que pour (1) et (2). Les résultats obtenus (fig. 3) confirment les résultats précédents. L'efficacité de l'iode à $25 \mathrm{mg} / \mathrm{l}$ est totale à $\mathrm{pH}$ 5,$3 ; 6,3$ et 7 , et chute à $\mathrm{pH} 8$ et 9 . Le rendement de fécondation reste inférieur à $10 \%$ jusqu'à $\mathrm{pH} 8$ pour atteindre $68 \%$ à $\mathrm{pH} 9$. 


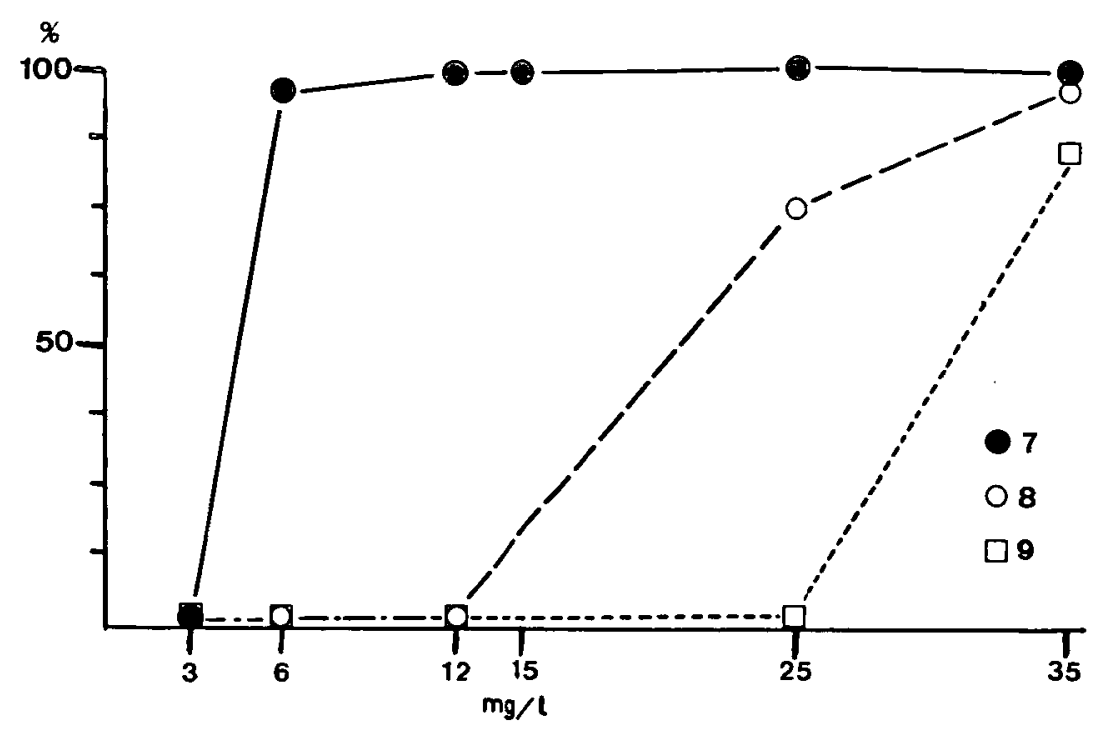

Figure 2 : Efficacité de l'iode in vitro sur le virus de la NPI. Une suspension virale de titre connu a été mélangée à des solutions tamponnées à $3 \mathrm{pH}$ (7-8-9) à l'aide de tétraborate de sodium, et contenant de l'iode aux concentrations indiquées. Après une incubation de $1 \mathrm{mn}$ l'iode était neutralisé par le thiosulfate de sodium, puis le virus résiduel titré par la technique des plages sous agarose. Les résultats sont exprimés en pourcentage d'inactivation par rapport à un témoin non traité.

Figure 2 : In vitro efficacy of iodine against IPN virus. A viral suspension of known titre was mixed with solutions buffered at $\mathrm{pH} 7,8$ or 9 with sodium tetraborate, and containing iodine at the indicated concentrations. After a $1 \mathrm{mn}$ incubation iodine was neutralized by sodium thiosulfate, and residual virus titrated by the plaque technique under agarose. The results are given as inactivation percentage calculated by comparison with a control virus suspension.

\section{Evaluation de l'innocuité de l'iode sur des spermes individuels}

Les ovules de 5 femelles répartis en 20 lots homogènes de $20 \mathrm{~g}$ (soit après comptage $238 \pm 8,4$ ovules, extrêmes $=223$ et 259 ) ont été fécondés par $0,3 \mathrm{ml}$ du sperme d'un seul mâle selon la technique indiquée sur le flacon de Romeiod. Dix mâles différents ont été utilisés. Les cinq premiers avaient été pressés régulièrement chaque semaine pendant un mois avant utilisation, les cinq derniers n'avaient pas été pressés depuis le début de la spermiation. Pour chaque mâle un premier lot (a) était inséminé en présence de DIA, un second (b) avec une solution iodée $(25 \mathrm{mg} / \mathrm{l})$ à $\mathrm{pH} 8$. Les mobilités étaient contrôlées individuellement au microscope et les spermatozoïdes comptés à la cellule de Malassez. La protéine de membrane $42 \mathrm{kDa}$ était titrée dans les liquides séminaux. Les rendements de fécondation (pourcentage d'oeufs "oeillés" à $250^{\circ} \mathrm{X}$ jours) se sont répartis entre 82 et $89,5 \%$ pour les fécondations pratiquées avec le DIA et entre 1,3 et $31 \%$ avec la solution iodée (tableau I). Exprimés en pourcentage du témoin DIA, les résultats vont de 1,6 à $37 \%$, sans corrélation avec la concentration du sperme en spermatozoïdes ni avec la concentration du liquide séminal en protéine $42 \mathrm{kDa}$. 


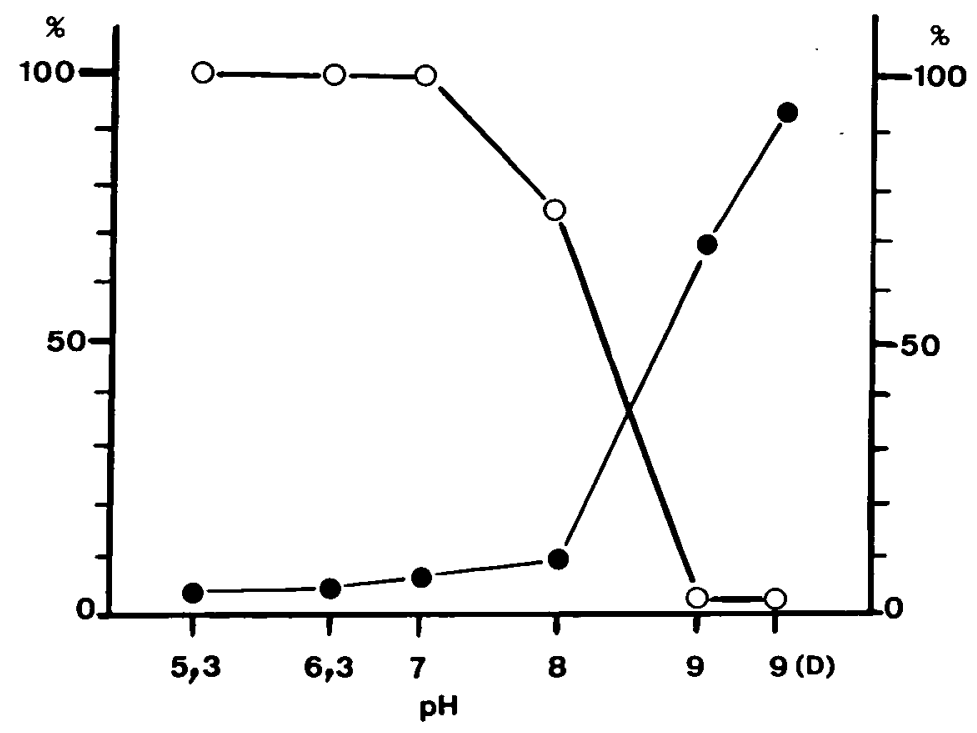

Figure 3 : Evaluation simultanée de l'innocuité et de l'efficacité d'une préparation à $25 \mathrm{mg} / \mathrm{l}$ d'iode actif à différents $\mathrm{pH}: 5,3$ (eau distillée), 6,3, 7, 8, 9 (tampon au tétraborate de sodium) et en dilueur d'insémination tamponné au trisglycine (D). Innocuité : des lots de 220 ovules ont été recouverts dé $30 \mathrm{ml}$ de milieu de fécondation. Le sperme $(0,3 \mathrm{ml})$ était ajouté immédiatement et le mélange effectué par transvasement. Les résultats sont exprimés en pourcentage d'oeufs embryonnés (๑--.--๑). Efficacité : une suspension de virus de la NPI était mélangée aux différentes solutions et incubée $1 \mathrm{mn}$ avant neutralisation de l'iode par le thiosulfate de sodium, puis le virus résiduel était titré par la technique des plages sous agarose. Les résultats sont exprimés sous forme de pourcentage d'inactivation par rapport à un témoin nọn traité (O----O)

Figure 3 : :Simültaneous asses sment of safety and efficacy of a $25 \mathrm{mg} / \mathrm{l}$ iodine solutión at pH 5.3 (distilled water), 6.3, 7, 8, 9 (sodium tetraborate buffer) and in a commercial insemination medium buffered at $\mathrm{pH} 9$ with trisglycine (D). Safety : groups of 220 ova were covered with $30 \mathrm{ml}$ of fecundation solution. Milt $(0.3 \mathrm{ml})$ was added immediately and rapidly mixed. The results are expressed as the percentage of eyed eggs (•-.--@). Efficacy : a suspension of IPN virus was diluted in the different solutions and incubated $1 \mathrm{mn}$ before iodine was neutralized by sodium thiosulphate. Remaining virus was titrated by the plaque technique under agarose. The results are expressed as the percentage of inactivation by comparison with a non-treated control (O----O).

\section{Essais de préincubation des spermatozoïdes avec une solution iodée}

Parallèlement aux essais dont les résultats ont été rapportés ci-dessus, des essais de préincubation courte ou longue des spermatozoïdes avec la solution iodée ont été tentés, avec l'idée qu'il s'agissait de la méthode la plus sûre pour inactiver le virus. La préincubation courte représentait une variante des protocoles de fécondations décrits cidessus. $0,5 \mathrm{ml}$ de sperme était mélangé rapidement à. $25 \mathrm{ml}$ du milieu de fécondation immédiatement versé sur les ovules égouttés. Dạns ces conditions, le rendement de fécondation est nul aux $\mathrm{pH} 6,3,7$ et 8 , pour atteindre $45 \%$ à pH 9 (tableau II). 
Tableau I : Rendements de fécondations (en \% d'oeufs embryonnés) obtenus en inséminant des lots de $\mathbf{2 4 0}$ ovules par des spermes individuels. Les ovules étaient recouverts par $30 \mathrm{ml}$ de DIA 532 ou de solution d'iode à $25 \mathrm{mg} / \mathrm{l}$ en eau de ville à $\mathrm{pH} 8$, avant d'ajouter $0,3 \mathrm{ml}$ de sperme. Les mâles 1 à 5 avaient été pressés régulièrement chaque semaine avant utilisation.

Table I : Fecundation rates (\% of embryonated eggs) obtained following insemination of 240 ova groups with individual milts. The ova were covered with $30 \mathrm{ml}$ of commercial insemination solution, or by a $25 \mathrm{mg} / \mathrm{l}$ iodine solution ( $\mathrm{pH} 8$ ), before $0.3 \mathrm{ml}$ of milt was added. Sires 1 to 5 had been stripped weekly before use.

\begin{tabular}{|c|c|c|c|c|l|l|l|l|}
\hline No & \multicolumn{3}{|c|}{ RENDEMENT DE FÉCONDATION } & \multicolumn{2}{l|}{ PROTÉINE 42 kDA } & \multicolumn{2}{|c|}{$\begin{array}{l}\text { CONCENTRATION } \\
\text { EN } \\
\text { SPERMATOZOÏDES }\end{array}$} \\
\hline & DIA & IODE & $\begin{array}{l}\text { IODE/ } \\
\text { DIA } \%\end{array}$ & RANG & $\%$ & RANG & $\begin{array}{l}\text { Spz/ml } \\
\times 10^{10}\end{array}$ & RANG \\
\hline 1 & 84 & 6,6 & 7,8 & 8 & 1,4 & 4,5 & 1,4 & 9,5 \\
\hline 2 & 82 & 1,3 & 1,6 & 10 & 1,4 & 4,5 & 3,7 & 2 \\
\hline 3 & 83 & 24 & 29 & 3 & 3,3 & 10 & 4,9 & 1 \\
\hline 4 & 86 & 6,4 & 7,5 & 9 & 0,3 & 1 & 3,2 & 4,5 \\
\hline 5 & 83 & 15 & 17,9 & 6 & 2 & 8 & 1,4 & 9,5 \\
\hline 6 & 90 & 23 & 25,6 & 4 & 0,7 & 2 & 3,4 & 3 \\
\hline 7 & 82 & 11 & 13,9 & 7 & 1,9 & 7 & 2,5 & 6,5 \\
\hline 8 & 84 & 31 & 37 & 1 & 0,8 & 3 & 3,2 & 4,5 \\
\hline 9 & 86 & 25 & 29,4 & 2 & 2,1 & 9 & 2,5 & 6,5 \\
\hline 10 & 85 & 21 & 24,9 & 5 & 1,5 & 6 & 1,7 & 8 \\
\hline
\end{tabular}

Tableau II : Résultat de fécondations pratiquées en mélangeant rapidement $0,5 \mathrm{ml}$ de sperme à $25 \mathrm{ml}$ de milieu de fécondation avant d'en recouvrir des lots de 220 ovules. Le rendement est exprimé en \% d'oeufs embryonnés.

Table II : Results of fecundations made by mixing rapidly $0.5 \mathrm{ml}$ of milt to $25 \mathrm{ml}$ of the indicated fecundation medium before pouring it on groups of 220 ova. The results are expressed as eyed eggs percentage.

\begin{tabular}{|c|c|c|c|c|c|c|c|c|c|c|c|}
\hline $\begin{array}{c}\text { MILIEU DE } \\
\text { FÉCONDATION }\end{array}$ & $\begin{array}{c}\text { DIA } \\
532\end{array}$ & \multicolumn{2}{|c|}{$\begin{array}{c}\mathrm{H}_{2} \mathrm{O} \\
\text { distillée }\end{array}$} & \multicolumn{6}{|c|}{ TAMPON TÉTRABORATE } \\
\hline $\mathrm{pH}$ & 9 & \multicolumn{2}{|c|}{5,3} & \multicolumn{2}{|c|}{6,3} & \multicolumn{2}{|c|}{7} & \multicolumn{2}{|c|}{8} & \multicolumn{2}{c|}{9} \\
\hline lode mg/l & 0 & 0 & 25 & 0 & 25 & 0 & 25 & 0 & 25 & 0 & 25 \\
\hline $\begin{array}{c}\text { Pourcentage } \\
\text { de fécondation }\end{array}$ & 92 & 89 & 0 & 82 & 0 & 80 & 0 & 89 & 0 & 70 & 45 \\
\hline
\end{tabular}


La préincubation «longue» consistait en une incubation de $1 \mathrm{mn}$ dans une solution saline tamponnée au tétraborate additionnée de $2 \mathrm{~g} / \mathrm{l}$ de $\mathrm{KCl}$ afin d'éviter l'activation prématurée des spermatozoïdes. Ce sperme dilué était ensuite déposé sur les lots de 200 ovules et l'insémination déclenchée par l'addition de $50 \mathrm{ml}$ de DIA. L'emploi de $0,5 \mathrm{ml}$ de sperme pour $5 \mathrm{ml}$ de solution de $\mathrm{KCl}$ a conduit à des rendements supérieurs à $60 \%$ pour les trois $\mathrm{pH}$ (7-8-9) et les trois concentrations d'iode (15-25-35 mg/l) employés (tableau III). Par contre, quand la concentration du sperme était divisée par 5-(soit 1/50) les rendements chutaient brutalement. II était intéressant de déterminer en parallèle l'efficacité de l'iode contre le virus en présence de sperme. Une suspension virale de titre connu a été incubée $1 \mathrm{mn}$ avec une solution d'iode à $25 \mathrm{mg} / \mathrm{l}$ additionnée de sperme à $\mathrm{pH} 7$. Pour une concentration en sperme de 1/250 la neutralisation du virus esṫ totale, pour devenir nulle à $1 / 50$ et $1 / 10$ (tableau IV).

Tableau III : Résultats de fécondations pratiquées en incubant le sperme $(0,1$ ou $0,5 \mathrm{ml}$ ) dans $5 \mathrm{ml}$ de solutions tamponnées contenant $2 \mathrm{~g} / \mathrm{l}$ de $\mathrm{KCl}$ et de l'iode aux concentrations indiquées pendant $1 \mathrm{mn}$ avant d'en recouvrir les lots de $\mathbf{2 0 0}$ ovules et d'ajouter $50 \mathrm{ml}$ de DIA 532.

Table III : Results of inseminations performed by incubating milt $(0.1$ or $0.5 \mathrm{ml})$ in $5 \mathrm{ml}$ of buffered solutions containing $2 \mathrm{~g} / \mathrm{K} \mathrm{KCl}$ and iodine at the indicated concentrations during $1 \mathrm{mn}$ before pouring it on groups of 200 ova and adding $50 \mathrm{ml}$ of commercial insemination buffer.

\begin{tabular}{|c|c|c|c|c|c|c|c|c|c|c|c|c|c|}
\hline $\begin{array}{c}\text { Milieu } \\
\text { d'incubation }\end{array}$ & DIA & \multicolumn{12}{|c|}{ Tampon Tétraborate } \\
\hline $\mathrm{pH}$ & 9 & \multicolumn{4}{|c|}{7} & \multicolumn{4}{|c|}{8} & \multicolumn{4}{|c|}{9} \\
\hline $\begin{array}{l}\text { lode } \\
\mathrm{mg} / \mathrm{l}\end{array}$ & 0 & 0 & 15 & 25 & 35 & 0 & 15 & 25 & 35 & 0 & 15 & 25 & 35 \\
\hline $\begin{array}{c}\text { Rendement } \\
\text { (sperme au } \\
1 / 10 \text { ) }\end{array}$ & 70 & 74 & 75 & 65 & 61 & 80 & 72 & 71 & 62 & 77 & 70 & 76 & 70 \\
\hline $\begin{array}{c}\text { Rendement } \\
\text { (sperme au } \\
1 / 50 \text { ) }\end{array}$ & 80 & 78 & 3 & 0 & 0 & 79 & 0 & 0 & 0 & 79 & 95 & 11 & 0 \\
\hline
\end{tabular}

Tableau IV : Inactivation du virus de la N.P.I. par l'iode à $25 \mathrm{mg} / \mathrm{l}$ à pH 7 en présence de sperme, après une incubation de $1 \mathrm{mn}$.

Table IV : Inactivation of IPN virus by $25 \mathrm{mg} / \mathrm{l}$ iodine at $\mathrm{pH} 7$ with different concentrations of milt.

\begin{tabular}{|c|c|c|c|c|}
\hline $\begin{array}{c}\text { Concentration } \\
\text { du sperme }\end{array}$ & 0 & $1 / 250$ & $1 / 50$ & $1 / 10$ \\
\hline $\begin{array}{c}\% \text { d'inactivation } \\
\text { du virus }\end{array}$ & 100 & 100 & 0 & 0 \\
\hline
\end{tabular}




\section{Influence du tri des oeufs sur la transmission}

Deux expériences ont été réalisées successivement, comportant chacune deux lots de 2000 ovules issus de 4 femelles (1) et de 6 femelles (2) et utilisant les spermes de 8 mâles (1) et de 6 mâles (2). Le virus de la NPI concentré au Polyéthylène glycol était repris dans $9 \mathrm{ml}$ de DIA additionné de $\mathrm{KCl} 2 \mathrm{~g} / \mathrm{l} .1 \mathrm{ml}$ de sperme y était ajouté, la concentration en spermatozoïdes du sperme étant $4 \times 10^{10 / \mathrm{ml}}$ (1) et $1,2 \times 10^{10 / \mathrm{ml}}$ (2). Le titre du virus était de $1,8 \times 10^{10}$ u.f.p/ml (1) et $2,5 \times 10^{10}$ u.f.p/ml (2) ce qui donnait un rapport particules virales infectieuses/spermatozoïdes égal à 4 (1) et 18 (2). Après $10 \mathrm{mn}$ d'incubation $5 \mathrm{ml}$ de cette suspension étaient déposés sur les ovules, et l'insémination déclenchée par addition de $75 \mathrm{ml}$ de DIA. Après durcissement $(45 \mathrm{mn})$, tous les oeufs étaient désinfectés au Romeiod à $100 \mathrm{mg} / \mathrm{l}$ d'iode actif pendant $10 \mathrm{mn}$. Les deux lots étaient incubés dans des aquariums séparés. Les lots $A$ étaient triés régulièrement et les oeufs "blancs" éliminés immédiatement. A $250^{\circ} \mathrm{X}$ jours un tri «définitif» soigneux a conduit à l'élimination des oeufs non développés “jaunes" et des oeufs à embryogenèse manifestement imparfaite «jaunes à petits yeux", tandis qu'une seconde désinfection par le Romeiod à $100 \mathrm{mg} / \mathrm{l}$ d'iode était pratiquée. Les lots $B$ étaient laissés sans le moindre tri jusqu'à la fin de la résorption de la vésicule vitelline. Des oeufs éliminés des lots $1 \mathrm{~A}$ et $2 \mathrm{~A}$ ont été broyés individuellement pour recherche du virus. Le virus a pu être mis en évidence dans 11/16 oeufs blancs, 25/40 oeufs «jaunes" et 13/40 “jaunes à petits yeux". Des diagnostics virologiques pratiqués en fin de résorption sur des lots de 10 alevins apparemment normaux ont permis la mise en évidence du virus dans tous les lots, et la NPI clinique s'est manifestée dès la prise de nourriture (tableau V).

Tableau V : Transmission expérimentale de la N.P.I. par l'oeuf grâce à une préincubation des spermatozoïdes $10 \mathrm{mn}$ avec le virus $(1 \mathrm{ml}$ de sperme dans $9 \mathrm{ml}$ de suspension virale en DIA KCl $2 \mathrm{~g} / \mathrm{l}) .5 \mathrm{ml}$ de sperme dilué étaient déposés sur chaque lot de 2000 ovules avant d'ajouter $75 \mathrm{ml}$ de DIA 532. Les lots (A) étaient débarrassés au fur à mesure des oeufs blancs, et triés soigneusement à 250 degrés $X$ jours tandis que les lots (B) n'étaient pas triés.

Table V : Experimental IPNV egg-associated transmission achieved by preincubating sperms $10 \mathrm{mn}$ with the virus $(1 \mathrm{ml}$ milt in $9 \mathrm{ml}$ of concentrated virus resuspended in commercial insemination solution with $2 \mathrm{~g} / \mathrm{l} \mathrm{KCl}$ ). $5 \mathrm{ml}$ of diluted milt was then poured on each batch of 2000 ova and $75 \mathrm{ml}$ of commercial insemination solution were added. Groups (A) were cleared of dead eggs and sorted carefully at 250 degrees $X$ days while groups $(B)$ were not sorted.

\begin{tabular}{|l|c|c|c|c|}
\hline & \multicolumn{2}{|c|}{ Expérience 1 } & \multicolumn{2}{c|}{ Expérience 2 } \\
\hline Lots expérimentaux & $1 \mathrm{~A}$ & $1 \mathrm{~B}$ & $2 \mathrm{~A}$ & 2B \\
\hline ufp/spz & 4 & 4 & 18 & 18 \\
\hline Tri des oeufs au fur et à mesure & + & - & + & - \\
\hline Oeufs blancs de 0 à 25 j & 29 & & 80 & \\
\hline Jaunes non développés & 67 & & 203 & \\
\hline Jaunes « à petits yeux » & 50 & & 35 & \\
\hline 2ème désinfection Romeiod & + & - & + & - \\
\hline Isolement du virus & + & + & + & + \\
\hline N.P.I. clinique & + & + & + & + \\
\hline
\end{tabular}




\section{DISCUSSION ET CONCLUSION}

La "fécondation à l'iode» a suscité de grands espoirs et provoqué aussi maintes désillusions. II était donc important de procéder à une évaluation aussi rigoureuse que possible tant de son innocuité que de son efficacité, dans. la mesure où les informations issues des travaux et des observations en salmoniculture apparaissaient contradictoires. Dé cette éväluation ont été d'emblée éliminés les protocoles apparentés à la fécondation "classique", c'est-à-dire au cours desquels le sperme est déposé sur les ovules égouttés avant de recouvrir ceux-ci de la solution iodée : en effet des spermatozoïdes peuvent être activés par des traces de liquide coelomique et pénétrer dans l'ovule avant désinfection. Le choix du protocole diffusé auprès des éleveurs par le fournisseur de l'iodophore comme point de départ était logique. L'influence du pH sur l'action de l'iode est bien connu, et aussi son affinité pour différentes molécules organiques, (entre autres, les protéines) qui conduit à son inactivation (AMEND et PIETSCH, 1972 ; ELLIOTT et AMEND, 1978 ; $\bar{A}$ HNE et HELD, 1980). Comme les auteurs précités, nous ne nous sommes pas préoccupés des aspects purement chimiques de ces variations d'activité pour nous concentrer sur le résultat biologique. En particulier, nous avons vérifié que l'activité, virucide de l'iode était abolie par deux des composants du DIA : le tris et le glycocolle (résultats non détaillés icii). L'emploi du tétraborate permettait d'explorer une gamme de $\mathrm{pH}$ suffisante tout en respectant cette activité. Les fécondations réalisées suivant le protocole «Romeiod» à des $\mathrm{pH}$ égaux ou inférieurs à 8 (cas de nombreuses salmonicultures, . en particulier dans les régions de massifs cristallins) ont donné des rendements de fécondation inférieurs à $10 \%$, même avec une dose de $15 \mathrm{mg} / \mathrm{l}$ d'iode, ce qui explique de nombreux échecs rencontrés sur le terrain. Le temps de contact $(1 \mathrm{mn})$ choisi pour mesurer l'activité virucide est de l'ordre de grandeur de la durée de mobilité du spermatozoïde, donc du temps de contact maximum possible avant sa pénétration dans le micropyle. L'efficacité de l'iode contre le virus de la NPI a suivi fidèlement son activité spermicide. Les résultats obtenus ici recoupent ceux obtenus auparavant avec des souches virales différentes et des coñditions elles aussi différentes par AMEND et PIETSCH (1972) et ELLIOT et AMEND (1978) qui concluaient que entre 16 et $32 \mathrm{mg} / \mathrm{l}$ d'iode étaient suffisants pour inactiver le virus en $5 \mathrm{mn}$ à $\mathrm{pH} 6,9$ et par DESAUTELS et MACKELVIE (1975) qui avaient besoin de $35 \mathrm{mg} / \mathrm{l} \mathrm{en} 5 \mathrm{mn}$ à un $\mathrm{pH}$ non précisé. Par contre, pour AHNE et HELD (1980), il avait fallu $50 \mathrm{mg} / \mathrm{l}$ d'iode pour inactiver totalement le virus en $20 \mathrm{mn}$ à $\mathrm{pH} 7,8$. II apparaît clairement d'après les résultats (Fig. 3) qu'il est impossible de concilier efficacité contre le virus $(\mathrm{pH} \leq 7)$ et innocuité vis-à-vis des spermatozoïdes $(\mathrm{pH}>8)$. Il est tentant de conclure que les exemples de réussite de la fécondation en présence d'iode enregistrés dans des eaux calcaires, à pH élevé, ou en présenence de liquide coelomique (CASTRIC, 1984), résultaient à la fois de l'inactivation de l'iode, et de la faible fréquence de transmission naturelle de la NPI déjà observée (DORSON, 1989). Bien que les protocoles employés aient été tout à fait différents, on peut rapprocher ces résultats de ceux de AHNE, KELLY et SCHLOTFELDT (1989), qui ont infecté des oeufs $30 \mathrm{mn}$ après fécondation, et les ont désinfectés $30 \mathrm{mn}$ plus tard sans trouver un compromis satisfaisant entre innocuité et efficacité. II était intéressant de déterminer si la "qualité" du sperme utilisé pouvait influencer le rendement de fécondation, en pratiquant 10 fécondations avec les spermes de mâles individuels dont cinq avaient été régulièrement vidés de leur sperme disponible tandis que les cinq autres n'avaient pas été pressés. La concentration d'une protéine de membrane de PM 42 kDa dans le liquide séminal est considérée comme révélatrice du vieillissement du sperme (MAISSE, PINSON et LOIR, 1988 ; MAISSE, 1990). Le nombre de spermatozoïdes par ml pouvait aussi être déterminant. En fait, si une gamme étalée de rendements a pu être observée suivant les spermes employés (de 1,6 à $37 \%$ ), ces performances n'ont pas pu être reliées à la concentration en protéine $42 \mathrm{kDa}$ ni à la concentration en spermatozoïdes. Une préincubation des spermatozoïdes dans la solution iodée avant insémination pouvait apparaître comme le procédé le plus sûr dụ point de vue de l'efficacité. En fait, ce procédé est apparu comme extrêmement défavorable : dans des conditions où l'iode garde son activité ( $\mathrm{pH}$ inférieur à 8 , sperme dilué) le taux de fécondation est nul. II a été vérifié que. lorsqu'une forte concentration en sperme permettait un taux de fécondation acceptable, le virus n'était pas inactivé. On retrouve ici le rôle joué vis-à-vis de l'iode par les protéines. L'efficacité de la transmission du virus par le sperme infecté in vitro (déjà réalisée en 1985 par DORSON et TORCHY mais pas en 1988 par SMAIL et MUNRO) a été confirmée ici, avec des concentrations en virus comparables à celles qui ont pu être observées sur des 
mâles infectés. L'élimination soigneuse des oeufs morts en cours d'incubation ainsi que celle des embryons incomplets n'a pas été suffisante pour couper la transmission. On ne peut pas exclure que le virus infecte l'embryon comme cela était postulé, mais il est certain qu'un oeuf peut rester infectieux après une désinfection externe et malgré un développement normal de l'embryon. II reste peu plausible qu'un embryon infecté puisse survivre, et il est probable que le virus reste adsorbé aux restes de la membrane du spermatozoïde, donc au niveau de l'espace périvitellin. Cette hypothèse redonne de l'intérêt aux pratiques de durcissement de l'oeuf en présence d'iode, déjà testées par AMEND (1974) du seul point de vue de l'innocuité, et qui mériteraient une étude plus complète. La rareté de la transmission interne rend assez facile d'éviter celle-ci en incubant et en élevant séparément les fratries issues de géniteurs infectés (WOLF, QUIMBY, CARLSON et BULLOCK, 1968 ; DORSON, résultats non publiés). On peut aussi pratiquer un diagnostic virologique sur des spermes individuels qui seront congelés en attendant le résultat. II faut préciser que c'est aujourd'hui la seule possibilité en médecine humaine pour lutter contre la transmission du virus du SIDA en insémination artificielle (SHERMAN, 1990, cité par SHERMAN, HOSTETLER, McHENRY et DALY, 1991). Cependant, des techniques de testage rapide du sperme quant à la présence du virus de la NPI pourraient écourter le temps de réponse suffisamment pour éviter la congélation (RODRIGUEZ SAINT-JEAN, PEREZ-PRIETO et VILAS MINONDO, 1992). Par contre, les dangers et l'inutilité de la fécondation en présence d'iode, quelle que soit la technique employée, ressortent bien de cette étude. Même en acceptant des taux de fécondation de l'ordre de $10 \%$, justifiés pour conserver des génotypes intéressants, un risque subsiste, mais qu'on peut éliminer par une quarantaine sérieuse. Ceci, bien sûr, n'enlève rien à l'intérêt de la désinfection des oeufs par l'iode en fin de durcissement, et au stade oeillé.

\section{REMERCIEMENTS}

Ce travail n'a pu être mené à bien que grâce à un financement par le Syndicat des Sélectionneurs Avicoles et Aquacoles Français, et les commentaires de P. RAULT sur le manuscrit ont été appréciés. Une grande partie a été réalisée dans les installations piscicoles expérimentales de Jouy-en-Josas tandis que les gamètes étaient prélevés à la pisciculture INRA de Gournay/Aronde avec la contribution des personnels de ces deux unités expérimentales. Les auteurs remercient Nicole DESCHAMPS pour la frappe du manuscrit.

\section{BIBLIOGRAPHIE}

AHNE W., 1983. Presence of infectious pancreatic necrosis virus in the seminal fluid of rainbow trout, Salmo gairdneri Richardson. Journal of Fish Diseases, (4), 377.

AHNE W., HELD C., 1980. Untersuchungen über die viruzide Wirkung von Actomar K30 auf fishpathogene Viren. Tierärztl. Umsch., 35, 308-318.

AHNE W., NEGELE R.D., 1985. Studies on the transmission of infectious pancreatic necrosis virus via eyed eggs and sexual products of salmonid fish. Fish and Shellfish Pathology (A.E. Ellis ed.), Academic Press, 261-269.

AHNE W., KELLY R.K., SCHLOTFELDT H.J., 1989. Factors affecting the transmission and outbreak of infectious pancreatic necrosis (IPN). Fish Health Protection Strategies (K. Lillelund and H. Rosenthal eds.), Federal Ministry for Research and Technology: 19-71.

AMEND D.F., 1974. Comparative toxicity of two iodophors to rainbow trout eggs. Transactions of the American Fisheries Society, 103 (1), 73-78.

AMEND D.F., PIETSCH J.P., 1972. Virucidal activities of two iodophors to salmonid viruses. J. Fish Res. Board Can., 29, 61-65.

BILLARD R., 1977. Utilisation d'un système tris-glycocolle pour tamponner le dilueur d'insémination pour truite. Bull. Fr. Piscic., 264, 102-112. 
BILLARD R., JALABERT B., 1974. L'insémination artificielle de la truite (Salmo gairdneri Richardson) II. Comparaison des effets de différents dilueurs sur la conservation de la fertilité des gamètes avant et après insémination. Ann. Biol. Anim. Bioch. Biophys., 14 (4A), 601-610.

BILLARD R., DE MONTALEMBERT G., 1976. Effet des iodophores sur les gamètes et les oeufs de truite arc-en-ciel. Bull. Fr. Piscic., 263, 41-44.

BOOTLAND L.M., DOBOS P., STEVENSON R.M.W., 1991. The IPNV carrier state and demonstration of vertical transmission in experimentally infected brook trout. Dis. Aquatic Org., $10(1), 13-21$.

BULLOCK G.L., RUCKER R.R., AMEND D., WOLF K., STUCKEY A.M., 1976. Infectious pancreatic necrosis : transmission with iodine treated and non treated eggs of brook trout (Salvelinus fontinalis). Journal of the Fisheries Research Board of Canada, 3 (53), 1197-1198.

CASTRIC J., 1984. La nécrose pancréatique infectieuse chez la truite arc-en-ciel. Généralités, transmission et moyens de lutte : résultats obtenus au LNPAA en 1983 et 1984. La Pisciculture Française, 77, 17-19.

DE KINKELIN P., SCHERRER R., 1970. Le virus d'Egtved. I. Développement, stabilité et structure de la souche danoise F1. Ann. Rech. Veter., 1, 17-30.

DESAUTELS D., MACKELVIE R.M., 1975. Practical aspects of survival and destruction of infectious pancreatic necrosis virus. Journal of the Fisheries Research Board of Canada, 32 (4), 523-531.

DOBOS P., HILL B.J., HALLETT R.; KELLS D.T., BECHT H., TENINGES D., .1979. Biophysical and biochemical characterization of five animal viruses with bisegmented double stranded RNA genomes. Journal of Virology, 32 (2), 593-605.

DORSON M., 1988. Vaccination against Infectious Pancreatic Necrosis. In ELLIS A.E., Fish vaccination, 162-171, Academic Press, London.

DORSON M., 1989. Point de vue sur l'efficacité et les limites de la «fécondation à l'iode» pour éviter la transmission de la nécrose pancréatique infectieuse des salmonidés. La Pisciculture Française, 95, 33-35.

DORSON M., TORCHY C., 1981. The influence of fish age and water temperature on mortalities of rainbow trout (Salmo gairdneri Richardson) caused by an european strain of infectious pancreatic necrosis virus. Journal of Fish Diseases, 4, 213-221.

DORSON M., TORCHY C., 1985. Experimental transmission of infectious pancreatic necrosis virus via the sexual products. Fish and Shellfish Pathology (A.E. ELLIS ed.), Academic Press, 251-260.

ECONOMON P.P., 1963. Experimental treatment of infectious pancreatic necrosis of brook trout with polyvinylpyrrolidone-iodine. Trans. Am. Fish Soc., 92, 180-182.

ELLIOT D.G., AMEND D.F., 1978. Efficacy of certain disinfectants against infectious pancreatic necrosis virus. Journal of Fish Biology, 12, 277-286.

FIJAN N.N., GIORGETTI G., 1978. Infectious pancreatic necrosis : isolation of virus from eyed eggs of rainbow trout, Salmo gairdneri Richardson. Journal of Fish Diseases, 1 (3), 269-270.

FRANTSI C., SAVAN M., 1971. Infectious pancreatic necrosis virus. Temperature and age factors in mortality. Journal of Wildlife Diseases, 7, 249-255.

HILL B.J., 1982. Infectious pancreatic necrosis virus and its virulence. Microbial Diseases of Fish (R.J. Roberts ed.), Academic Press, 91-114.

KLONTZ G.W., 1981. Désinfection des gamètes par l'iode pour prévenir la transmission de la NPI. Conférence au Syndicat des Pisciculteurs Salmoniculteurs de France (10/06/81). 
LAPIERRE J., LARRIVEE D., BERTHIAUME L., 1986. Influence of water temperature and fish age on mortality in brook trout (Salvelinus fontinalis) infected with infectious pancreatic necrosis virus (IPNV). Aquaculture, 59 (2), 81-92.

MAISSE G., 1990. Le sperme des salmonidés. Le point sur les connaissances. Applications à la salmoniculture. INRA Prod. Anim., 3 (3), 223-228.

MAISSE G., PINSON A., LOIR M., 1988. Caractérisation de l'aptitude à la congélation du sperme de truite arc-en-ciel (Salmo gairdneri) par des critères physicochimiques. Aquat. Living Resour., 1, 45-51.

MULCAHY D., PASCHO R.J., 1984. Adsorption to fish sperm of vertically transmitted fish viruses. Science, 225, 333-335.

RODRIGUEZ SAINT-JEAN S., PEREZ PRIETO S.I., VILAS MINONDO M.P., 1992. Flow cytometry analysis of infectious pancreatic necrosis virus attachment to fish sperm. Dis. Aquat. Org., 15, 153-156.

SHERMAN J.K., HOSTETLER T.L., McHENRY K., DALY J.J., 1991. Cryosurvival during cryopresenvation of human semen. Cryobiology, 28, 246.

SILIM A., ELAZHARY Y., LAGACE A., 1982. Susceptibility of trouts of different species and origins to various isolates of infectious pancreatic necrosis virus. Canadian Journal of Fisheries Aquatic Science, 39, 1580-1584.

SMAIL D.A., MUNRO A.L.S., 1988. Infectious pancreatic necrosis virus in atlantic salmon : transmission via the sexual products Viruses of lower vertebrates, $W$. Ahne and $E$. Kurstak eds., 292-301.

SPRENT P., 1992. Pratique des statistiques non-paramétriques. INRA, Paris, $294 \mathrm{p}$.

SNIESZKO S.F., WOOD E.M., YASUTAKE W.T., 1957. Infectious Pancreatic Necrosis in Trout. A.M.A. Arch. Pathol., 63, 229-233.

WOLF K., 1988. Fish viruses and fish viral diseases. Comstock Publishing Associates, Cornell University Press, Ithaca, New York : $476 \mathrm{p}$.

WOLF K., QUIMBY M. C., 1962. Established eurythermic line of fish cells in vitro. Science, 135, 1065-1066.

WOLF K., SNIESZKO S.F., DUNBAR C.E., PYLE E., 1960. Virus nature of infectious pancreatic necrosis in trout. Proceedings of the Society of Experimental Biology and Medicine, 104, 105-108.

WOLF K., QUIMBY M.C., BRADFORD A.D., 1963. Egg-associated transmission of IPN virus of trouts. Virology, 21 (3), 317-321.

WOLF K., QUIMBY M.C., CARLSON C.P., BULLOCK G.L., 1968. Infectious pancreatic necrosis : selection of virus-free stock from a population of carrier trout. Journal of the Fisheries Research Board of Canada, 25 (2), 383-391.

WOOD E.M., SNIESZKO S.F., YASUTAKE W.T., 1955. Infectious pancreatic necrosis in brook trout. A.M.A. Arch. Pathol., 60, 26-28. 